\section{Enhancing VTP}

Addition of androgen deprivation therapy (ADT) to vascular targeted photodynamic therapy (VTP) significantly improved tumour control and reduced total serum PSA levels in prostate cancer xenograft models in mice compared with either treatment alone, according to new research.

VTP with the photosensitizer padeliporfin is a focal therapy for patients with low-risk unilateral prostate cancer. Following intravenous administration, padeliporfin is activated by near-infrared laser light supplied by optic fibres introduced into malignant areas, which results in oxygen radical production, destruction of blood vessels, and eventual tumour necrosis.

In this new study, researchers first investigated whether VTP activated signalling pathways that might be targetable with additional agents. Transcriptome analysis of VTP-treated, androgen receptor (AR)-expressing LNCaP (LNCaP-AR) xenograft samples showed enrichments in gene sets related to HIF1A, VEGFR, and AR pathways. The team then tested whether inhibition of AR signalling might improve the effectiveness of VTP in LNCaP-AR and VCaP (which express AR variant 7) tumour mouse models, using animals that were deficient either in T cells or in both T cells and B cells to also test the role of possible VTP-induced immune responses. Combination treatment with degarelix and VTP resulted in significantly improved tumour growth control compared with either modality alone in all xenograft models. Furthermore, total serum PSA measurement in the LNCaP-AR model showed that strongest PSA reductions occurred in the combination treatment group.

The team also histologically assessed tumour samples for cell apoptosis and necrosis and found that tumours treated with VTP and degarelix seemed to have more extensive areas of apoptosis, according to DNA fragmentation staining using TUNEL.

These results in mice suggest that addition of ADT to VTP can improve tumour control compared with VTP alone, pending verification in a clinical setting.

Clemens Thoma

ORIGINAL ARTICLE Kim, K. et al. Androgen deprivation therapy potentiates the efficacy of vascular targeted photodynamic therapy of prostate cancer xenografts. Clin Cancer Res. https://doi.org/10.1158/1078-0432.CCR-17-3474 (2018)

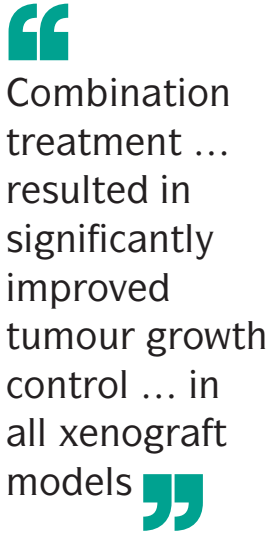

